\title{
Sustained Malaria Transmission despite Reactive Screen-and-Treat in a Low-Transmission Area of Southern Zambia
}

Kelly M. Searle, ${ }^{1 \star}$ Ben Katowa, ${ }^{2}$ Michael Musonda, ${ }^{2}$ Julia C. Pringle, ${ }^{3}$ Harry Hamapumbu, ${ }^{2}$ Japhet Matoba, ${ }^{2}$ Mukuma Lubinda, ${ }^{2}$ Timothy Shields, ${ }^{4}$ Tamaki Kobayashi, ${ }^{4}$ Jennifer C. Stevenson, ${ }^{2,3}$ Douglas E. Norris, ${ }^{3}$ Philip E. Thuma, ${ }^{2,3}$ Amy Wesolowski, ${ }^{4}$ and William J. Moss ${ }^{3,4}$ for the Southern and Central Africa International Center of Excellence for Malaria Research

${ }^{1}$ Division of Epidemiology and Community Health, University of Minnesota School of Public Health, Minneapolis, Minnesota; ${ }^{2}$ Macha Research Trust, Macha, Zambia; ${ }^{3}$ W. Harry Feinstone Department of Molecular Microbiology and Immunology, Johns Hopkins Bloomberg School of Public Health, Baltimore, Maryland; ${ }^{4}$ Department of Epidemiology, Johns Hopkins Bloomberg School of Public Health, Baltimore, Maryland

\begin{abstract}
Malaria elimination strategies are designed to more effectively identify and treat infected individuals to interrupt transmission. One strategy, reactive screen-and-treat, starts with passive detection of symptomatic cases at health facilities. Individuals residing within the index case and neighboring households are screened with a malaria rapid diagnostic test (RDT) and treated if positive. However, it is unclear to what extent this strategy is effective in reducing transmission. Reactive screen-and-treat was implemented in Choma district, Southern Province, Zambia, in 2013, in which residents of the index case and neighboring households within $140 \mathrm{~m}$ were screened with an RDT. From March 2016 to July 2018, the screening radius was extended to $250-\mathrm{m}$, and additional follow-up visits at 30 and 90 days were added to evaluate the strategy. Plasmodium falciparum parasite prevalence was measured using an RDT and by quantitative PCR (qPCR). A 24-single nucleotide polymorphism molecular bar-code assay was used to genotype parasites. Eighty-four index case households with 676 residents were enrolled between March 2016 and March 2018. Within each season, parasite prevalence declined significantly in index households at the 30-day visit and remained low at the 90-day visit. However, parasite prevalence was not reduced to zero. Infections identified by qPCR persisted between study visits and were not identified by RDT. Parasites identified within the same household were most genetically related; however, overall parasite relatedness was low and similar across time and space. Thus, despite implementation of a reactive screen-and-treat program, parasitemia was not eliminated, and persisted in targeted households for at least 3 months.
\end{abstract}

\section{INTRODUCTION}

Over the past two decades, there has been a decline in malaria incidence, morbidity, and mortality in more than 30 endemic countries, with eight formally transitioning from control to elimination strategies. ${ }^{1,2}$ However, a remaining challenge to elimination is identifying and treating all infected individuals (including asymptomatic and minimally symptomatic as well as symptomatic individuals) who may not seek care and treatment at health facilities but contribute to ongoing transmission. ${ }^{3-5}$ In low-transmission settings, these individuals may represent an important reservoir of transmission and present an obstacle to elimination. ${ }^{5-8}$ Strategies have been designed to identify and treat this reservoir through active case detection or mass drug administration. ${ }^{9-12}$ Active case detection strategies can be proactive, testing and treating individuals based on their membership in a specific risk group (e.g., school-age children), ${ }^{6,12,13}$ or reactive, by identifying transmission foci based on the diagnosis of symptomatic individuals. ${ }^{14-16}$ For reactive screen-and-treat, residents in the home of a confirmed symptomatic index case, and often their neighbors within a predefined radius, are screened with a rapid diagnostic test (RDT) and treated if positive. ${ }^{14,16,17}$ Reactive focal drug administration, by contrast, does not include screening, and instead, household residents are treated presumptively..$^{9,18,19}$ An advantage of focal drug administration is that individuals with sub-patent infections, those below the limit of detection by an RDT, are treated. However, this strategy limits the program's ability to

*Address correspondence to Kelly M. Searle, Division of Epidemiology and Community Health, University of Minnesota School of Public Health, 1300 S. 2nd St. Suite 300, Minneapolis, MN 55455. E-mail: ksearle@umn.edu accurately document the number of infected individuals within a community and requires a larger supply of antimalarials.

Zambia has had a dramatic decline in malaria cases in parts of the country over the past two decades, and Southern Province is considered a pre-elimination setting. ${ }^{20}$ Current (since 2013) surveillance and elimination strategies use volunteer community health workers (CHWs) to perform reactive screen-and-treat for confirmed index cases who have no history of travel outside the district. ${ }^{14}$ Members of the index and neighboring households within a 140-m radius are tested with an histidine-rich protein 2 (HRP-2)-based RDT, regardless of fever or other symptoms, within 1 week and treated if positive. However, it is unclear to what extent reactive screenand-treat is effective in further reducing transmission and eliminating the parasite reservoir. ${ }^{9,17,19,21-23}$

To assess the spatial and temporal effectiveness of reactive screen-and-treat in a low-transmission setting within the catchment area of Macha Hospital in Choma district, Southern Province, Zambia, the screening radius was extended to $250 \mathrm{~m}$, and follow-up visits were added at 30 and 90 days. The 250-m radius was chosen based on a previous study where $92 \%$ of cases were detected within this range. ${ }^{19}$ Plasmodium falciparum parasite prevalence was measured by quantitative PCR (qPCR) as well as RDT to detect low-level parasitemia. A 24-single nucleotide polymorphism (SNP) molecular barcode assay was used to genotype parasites, calculate genetic relatedness over space and time, and measure genetic distance between successive or persistent infections.

\section{METHODS}

Study site and population. This study was conducted in the rural catchment area of Macha Hospital in Choma district, 
Southern Province, Zambia, a low-transmission setting typical of southern Zambia. The region has a tropical savannah climate with a rainy season from December to April, followed by a cool dry season from May to August, and a hot dry season from September to November. ${ }^{24}$ The primary malaria vector is Anopheles arabiensis, which peaks during the rainy season, and malaria is almost exclusively due to $P$. falciparum. ${ }^{25}$ Plasmodium falciparum parasite prevalence measured by PCR declined from $9.2 \%$ in 2008 to $1 \%$ in $2013 .{ }^{26}$ Passively detected cases at health centers have since remained at historically low levels but with some variability, such as an increase in cases in 2016. ${ }^{19,20}$ Case management with artemisinin-based combination therapy was introduced in 2004, and insecticide-treated nets (ITNs) were widely distributed in 2007 and have been redistributed approximately every 3 years. $^{27,28}$

Study procedures. Enrollment began in March 2016 and continued through March 2018, with follow-up visits continuing through July 2018 . For this study, the reactive screenand-treat radius was expanded from 141 to $250 \mathrm{~m}$ of the index case household. Community health workers who performed reactive screen-and-treat sent a short message service text message to the study team at Macha Research Trust when an eligible index case was identified. A study team member and the $\mathrm{CHW}$ visited the index household for a notification visit during which the head of household and residents were informed that the $\mathrm{CHW}$ and study team would return the following day to perform the combined programmatic reactive screen-and-treat and study procedures. GPS coordinates for the index household were collected at the notification visit and mapped in ArcGIS v. 10 (ESRI, Redlands, CA). A Geo-Eye-1 high-resolution satellite image collected in 2011 (Apollo Mapping, Boulder, CO) was used to identify neighboring households eligible for screening at 140 - and $250-\mathrm{m}$ radii from the index households. The images were printed and used to guide the $\mathrm{CHW}$ for programmatic reactive screen-and-treat within $140 \mathrm{~m}$ and the study team for enrollment of eligible households up to $250 \mathrm{~m}$ from the index household.

All residents of index and neighboring households within $250 \mathrm{~m}$ were eligible for study enrollment. Written informed consent was obtained from adults, and parental permission was provided for children. At the initial visit, a questionnaire was administered to collect information on demographic characteristics, malaria knowledge and prevention, recent malaria symptoms (e.g., fever, chills, and headache), healthseeking behaviors, recent malaria treatment, and recent travel history. Temperature was collected using a tympanic thermometer. Finger prick blood samples were collected for a $P$. falciparum HPR-2 RDT (SD Bioline, Gyeonggi-do, Republic of Korea) and as a dried blood spot (DBS) on a filter paper (Whatman $903^{\mathrm{TM}}$ Protein Saver Card, Sigma-Aldrich, Piscataway, NJ) for molecular analysis. Individuals with a positive RDT, with the exception of the index case on the initial visit, were offered treatment with Coartem ${ }^{\circledR}$ (artemether plus lumefantrine, Novartis, Basel, Switzerland).

Study follow-up visits were conducted 30 and 90 days after the initial visit for all households. The study design was an open cohort, so residents in a household not present at a previous visit were invited to participate, offered informed consent, and enrolled at any follow-up visit. When enrolled households or participants were not present at the initial or follow-up visit, up to three visits were made. After these three attempts, if the household or participant was not available, the visit was treated as missing data. Similar study procedures were conducted at the follow-up visits as during the initial visit, with the exception that if the index case tested positive by RDT at a follow-up visit they were offered treatment with Coartem.

This study was approved by the Tropical Diseases Research Centre Ethics Review Committee and the Institutional Review Board at the Johns Hopkins Bloomberg School of Public Health.

Laboratory procedures. DNA was extracted from a single spot from the DBS card using a Chelex ${ }^{\odot}$ (Bio-Rad Laboratories, Hercules, CA) extraction protocol as previously described. ${ }^{26}$ Quantitative PCR was performed on all samples to detect the presence of the $P$. falciparum cytochrome b gene (Pfcytb). The limit of detection was determined to be one parasite per $\mu \mathrm{L}$ using standard genomic DNA dilution series and filter paper spotted with cultured parasites (NF54). Primers were designed to detect the presence of Pfcytb, and amplification was detected by fluorescence signal of SYBR ${ }^{\circledR}$ Green. Each reaction contained $5 \mu \mathrm{L}$ of DNA template, $5 \mu \mathrm{L}$ of SYBR Green PCR Master Mix (Thermo Fisher, Waltham, MA), and 200 nM of forward primer (5' CCT GAT AAT GCT ATC GTA 3') and reverse primer ( $5^{\prime}$ TAA TAC AAT TAC TAA ACC AGC $\left.3^{\prime}\right)$. All qPCR-positive samples were evaluated on a $4 \%$ agarose gel to confirm the product size of the amplicon. Only samples confirmed by both qPCR and gel electrophoresis were considered qPCR positive.

A 24-SNP molecular bar-code assay was run using a TaqMan protocol at Macha Research Trust (see Supplemental Data). The 24-SNP molecular bar code was developed to distinguish parasite populations using PCR-based technology in a field research setting. ${ }^{29}$ DNA was extracted from a second DBS for samples confirmed to be parasite positive by qPCR. Because of low parasite DNA concentrations, samples were pre-amplified before performing the 24-SNP molecular bar-code assay. Specific methods for the pre-amplification step were described elsewhere. ${ }^{30,31}$ For each of the 24-SNP assays, $2.0 \mu \mathrm{L}$ of pre-amplified sample DNA was added to $10 \mu \mathrm{L}$ of TaqMan master mix, $7.5 \mu \mathrm{L}$ distilled water, and $0.5 \mu \mathrm{L}$ TaqMan commercially available primer and probe assay mixture. ${ }^{29}$ For each assay, three known positive controls and two negative, no-template controls were run. Positive controls consisted of DNA samples from $P$. falciparum strains obtained from BEI Resources (Manassas, VA) with known haplotypes for all 24 SNPs. Typically, 12-SNP assays were run for five samples at a time with controls on a 96-well plate. The assays were run on the Applied Biosystems StepOnePlus ${ }^{\mathrm{TM}}$ (Thermo Scientific), and the Roche LightCycler 480 II $^{\mathrm{TM}}$ (Roche Diagnostics Corporation, Indianapolis, IN) real-time PCR systems. SNP calls were made automatically based on the highresolution melt curves using software programs accompanying the real-time PCR systems as one of the two alleles or mixed. In cases where SNP calls could not be made automatically, determination was made manually by the study investigators but was not systematic by allele. Otherwise, the SNP call was classified as failed. Samples with failed SNP calls were repeated up to three times. If they failed on all repeated assays, they were treated as missing data.

Statistical analyses. Characteristics of the study population were compared by household type (index households, neighbors within $140 \mathrm{~m}$, and neighbors between 141 and $250 \mathrm{~m}$ ) and study visit. The malaria transmission season was 
defined as October through the following September and was used rather than the calendar year to more accurately capture the seasonal peak in cases and transmission. Differences in median age, distance from different stream orders (using the Strahler classification system, in which two category 1 streams join to form a category 2 stream, two category two streams join to form a category three stream, and so on), normalized difference vegetation index, and elevation were estimated using a Kruskal-Wallis test. Differences in gender, presence of fever (defined as tympanic temperature $>38^{\circ} \mathrm{C}$ ), reported ITN use, recent travel, parasite prevalence by RDT, and parasite prevalence by qPCR were estimated using a Clopper-Pearson binomial exact test.

Parasite genetic analysis. Parasite bar coding was attempted on all qPCR-positive samples (see Data File 1). The distribution of parasite densities for all qPCR-positive samples was graphed and compared for samples that were successfully and not successfully bar-coded. Samples missing $>11$ of the 24 SNPs in the bar code were excluded from analysis. Pairwise agreement between all samples was calculated using a modified SNP $\Pi$ to account for mixed infections and missing data. ${ }^{30}$ Details of the calculation of the modified SNP $\Pi$ were described elsewhere. ${ }^{32}$ In brief, this was calculated as the pairwise percent agreement between the 24-SNP sequences for each of the samples. ${ }^{32}$ This resulted in a pairwise genetic relatedness matrix. Pairwise time difference and distance between household matrices were created for all samples.

Genetic distance between bar-code sequences was also calculated for those with analyzable bar codes. Genetic distance between bar-code sequences was compared and graphed across household types and between participants who were parasitemic on multiple visits and was not set to a specified threshold. The genetic distance was compared between consecutive infections to determine relatedness between infections to infer persistence or new infections between visits.

\section{RESULTS}

Characteristics of the study population. Eighty-four index households with 676 residents were enrolled and had an initial visit between March 2016 and March 2018 (Figure 1A). Enrollment decreased to 76 households with 544 residents on the 30-day visit and 75 households with 463 residents on the 90-day visit. One hundred and forty households with 675 residents were enrolled at the initial visit in neighboring households within $140 \mathrm{~m}$ of the index household, which decreased to 106 households with 438 residents and 111 households with 400 residents at the 30- and 90-day visits, respectively. One hundred forty-eight households with 864 residents were enrolled at the initial visit in neighboring households between 141 and $250 \mathrm{~m}$ of the index household, which decreased to 113 households with 574 residents and 99 households with 470 residents with follow-up data at the 30and 90-day visits, respectively (Table 1).

A map of the participating households showed clustering due to the study design (Figure 1A). The study population was young, with a median age of 12 years for all household types and visits, with the exception of index households at the 90day visit for which the median age was 11 years. The prevalence of fever was low and decreased significantly after the initial visit in all household types. Reported ITN use was low and increased slightly after the initial visit, but only in index households. The prevalence of recent travel also was low but was significantly higher in index households on the initial visit (9\% [7.0-11.5]) than in all other visits and household types. Environmental factors did not differ between index and neighboring households (Table 1).

Parasite densities for qPCR-positive samples were low, with a median of 17.4 (4.2-78.8) copies per $\mu \mathrm{L}$. Samples with successful bar-code sequences had a median of 19.7 (3.6-100.0) copies/ $\mu \mathrm{L}$, and those not successfully bar-coded had a median of 15.3 (4.2-63.0) copies/ $\mu \mathrm{L}$. Although parasite densities were slightly higher among samples that were successfully bar-coded, this was not statistically significant, and the distributions overlapped (Supplemental Figure S1).

Reactive screen-and-treat using an RDT did not capture most parasitemic individuals. Plasmodium falciparum prevalence as measured by both RDT and qPCR was low. The highest prevalence was in index households at the initial visit $(1.9 \%$ [1.0-3.3]) and decreased in all household types after the initial visit (Table 1, Figure 1B). Unsurprisingly, parasite prevalence measured by qPCR was significantly higher than that by RDT in all household types at each visit, ranging from 2 to 10.5 times higher (Figure 1B). In general, the sensitivity of the RDT was low, identifying only $20 \%$ of qPCR-positive individuals.

Reactive screen-and-treat reduced parasite prevalence but did not achieve elimination. Following reactive screen-andtreat, parasite prevalence by both RDT and qPCR declined at 30 and 90 days compared with the initial visit. The highest parasite prevalence was recorded in index households at the initial visit (7.9\% [6.0-10.2]) and decreased in all household types over follow-up with the exception of the 30-day visit in neighboring households between 141 and $250 \mathrm{~m}$ of the index household (Table 1, Figure 1B). When stratified by transmission season, parasite prevalence by qPCR showed similar decreasing trends by household type and follow-up visits (Figure 1C). Over the three transmission seasons, parasite prevalence was consistently highest in index households, with variability in prevalence over follow-up visits in all neighboring households. Parasite prevalence reached zero only in neighboring households between 141 and $250 \mathrm{~m}$ from index households on the 30 -day visit in 2016 as well as the initial and 90-day visits in 2018 , but was above zero for all other household types and visits (Figure 1D).

Few individuals were repeatedly positive with or without treatment. Only 145 participants had a positive RDT or qPCR result at any study visit. Thirty-seven participants (1.5\%) had a positive RDT result with only one being repeatedly positive on a subsequent follow-up visit (initial visit and the 90-day followup) (Figure 2A). This individual had a missing 30-day follow-up visit. Although more individuals were parasitemic by qPCR (148 events among 138 participants), only 10 participants were positive by qPCR a second time (Figure 2B). Nine of these repeated qPCR-positive results were on the initial and 30 -day visits with only one on the 30 - and 90 -day visits. None of these infections were identified by RDT on the initial visit, and only two were identified on the 30-day visit. Parasitemia identified on the 30-day visit by qPCR that persisted to the 90day visit was also not identified on either visit by RDT. Although these individuals who were positive only by qPCR were not treated, most were negative by qPCR at the 30-day or 90-day visit without becoming RDT positive. 
Parasites were related across time and clustered in space. Ideally, a reactive screen-and-treat program would identify not only asymptomatic infections but also those related by transmission to the index case. Genetic relatedness based on 24-SNP bar-code data was used as a proxy for transmission relatedness. Only 68 samples (45.9\%) had analyzable bar-code sequences from which genetic relatedness between infections was calculated, in part because of the low levels of parasitemia. Bar codes from parasites identified within the same household were most highly related (Figure $3 \mathrm{~A}$ ). However, there was no clear pattern between parasite relatedness in the index household and other households or between neighboring households. Relatedness decreased slightly with increasing time between detection (Figure 3B). However, parasites were related across seasons, as demonstrated by clustering of highly related parasites in three bands indicating the three transmission seasons (Figure 3B), suggesting some focal within-season parasite relatedness and maintenance of transmission with related parasites between seasons.

Interestingly, parasites were less related between an index case and a subsequent asymptomatic case identified as part of the reactive case detection event if the subsequent case was RDT positive (average genetic distance between index and RDT $+\& \mathrm{PCR}+=0.45$, index and PCR $+=0.32, P=0.05$ ), although this result was not significant (Figure $3 C$ ). This suggests that reactive screen-and-treat may not be capturing transmission-related infections.

A small number of persistent infections may impact transmission. Although the sample size was limited, the genetic distance between bar codes from individuals who had multiple qPCR-positive visits was analyzed. Only 10 individuals were qPCR positive on multiple visits. These 10 individuals resided in nine households and were collected during the 2016 transmission season. Of these 10 individuals, five had samples that were successfully bar-coded at two time points one month apart (no individuals with three PCR positive visits had three successful bar codes). For these five individuals, the genetic distance between these bar codes was calculated, and, for comparison, the genetic distance between bar codes collected 1 month apart also was estimated. Three individuals had bar codes that were highly related and above the expected genetic relatedness (Figure 3D). These three individuals
A

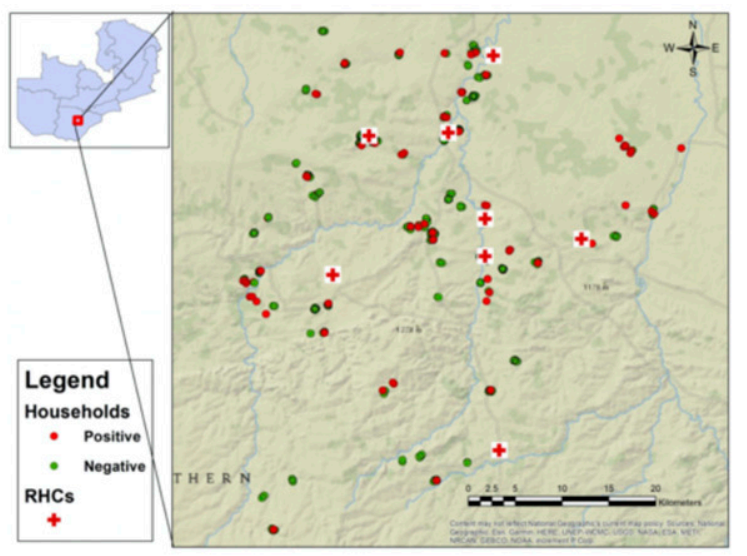

C
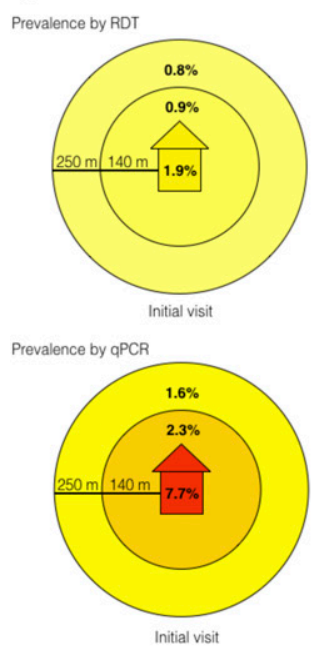
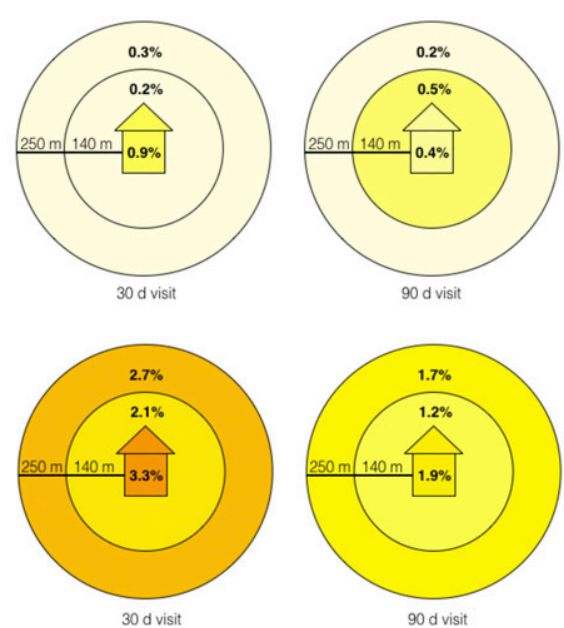

B

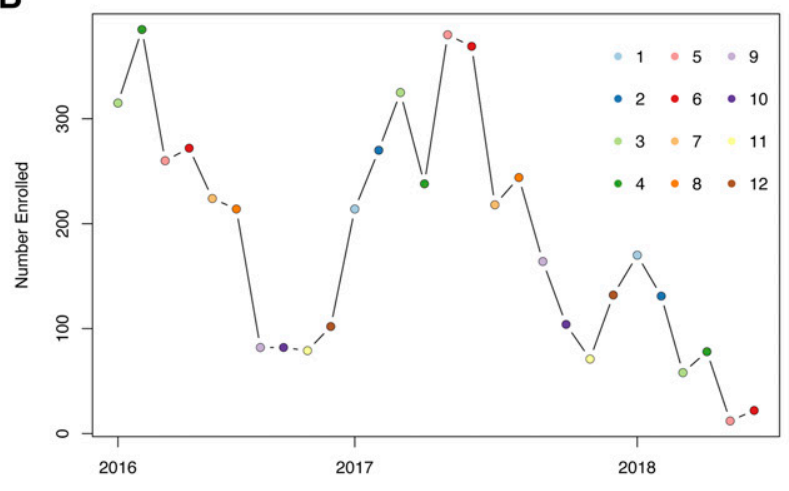

D

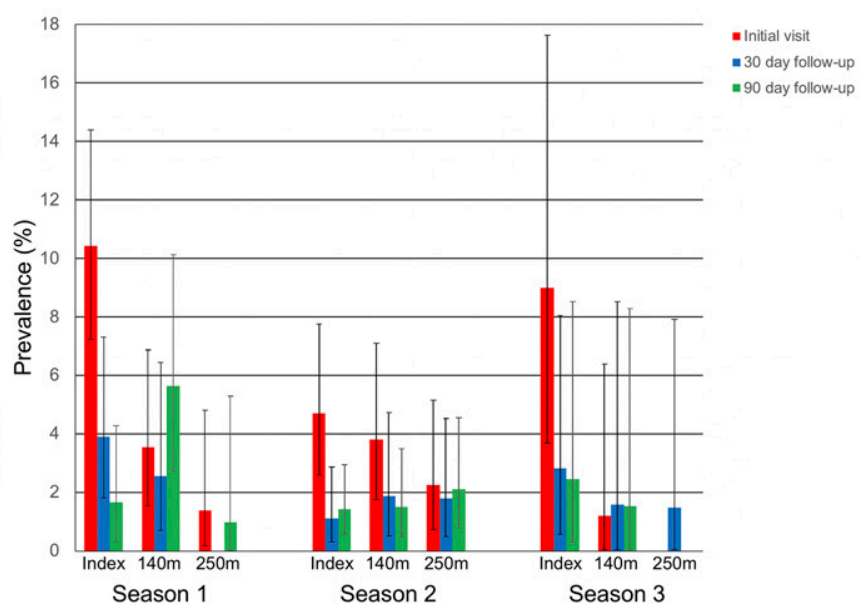

FIGURE 1. Study area and parasite prevalence of those sampled. (A) Location of all households sampled as part of the reactive screen-and-treat program within the catchment area of Macha Hospital within Choma district, Southern Province. Households are marked either positive (red) or negative (green) if at least one positive case (by quantitative PCR [qPCR]) was identified in that household. (B) Number of individuals enrolled in the study colored by month (1-12). (C) Parasite prevalence by rapid diagnostic test (RDT) (top row) and qPCR (bottom row) for the index household, households within $140 \mathrm{~m}$, and those between 141 and $250 \mathrm{~m}$. Tests were performed during initial, 30-day, and 90 -day visits. Overall prevalence by RDT was lower than that by GPCR and highest in the index household. (D) Prevalence by season for the index, $140 \mathrm{~m}$ and $250 \mathrm{~m}$ households by visit number. This figure appears in color at www.ajtmh.org. 


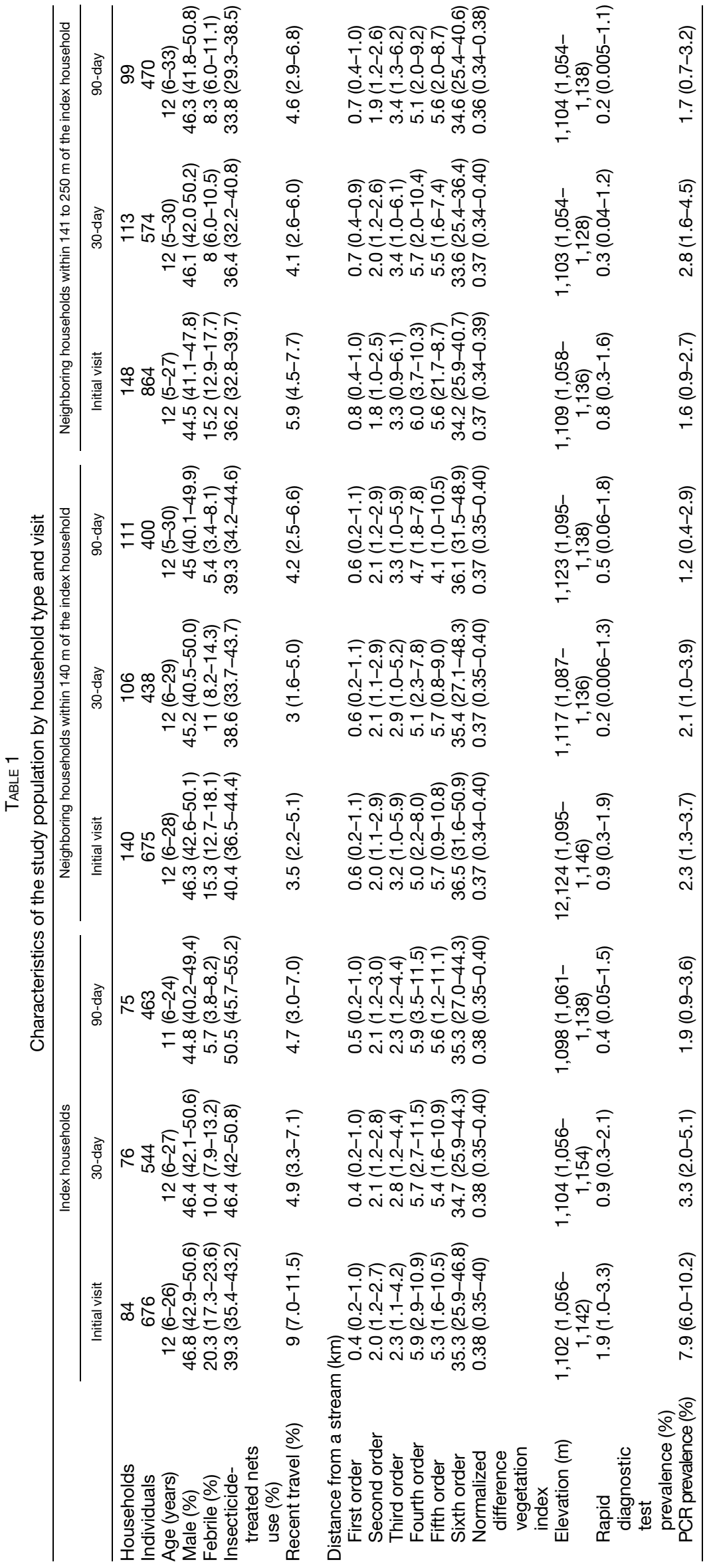


A

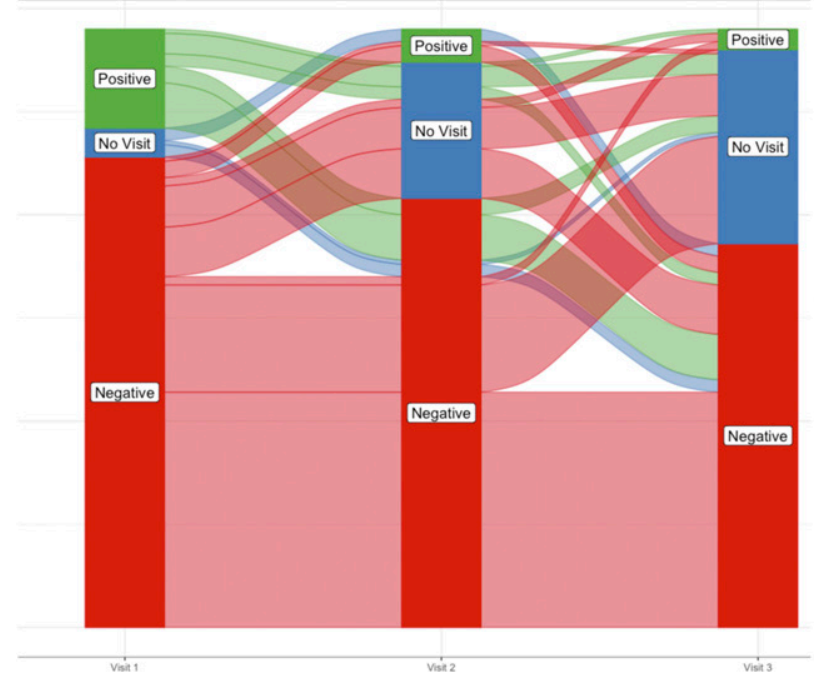

B

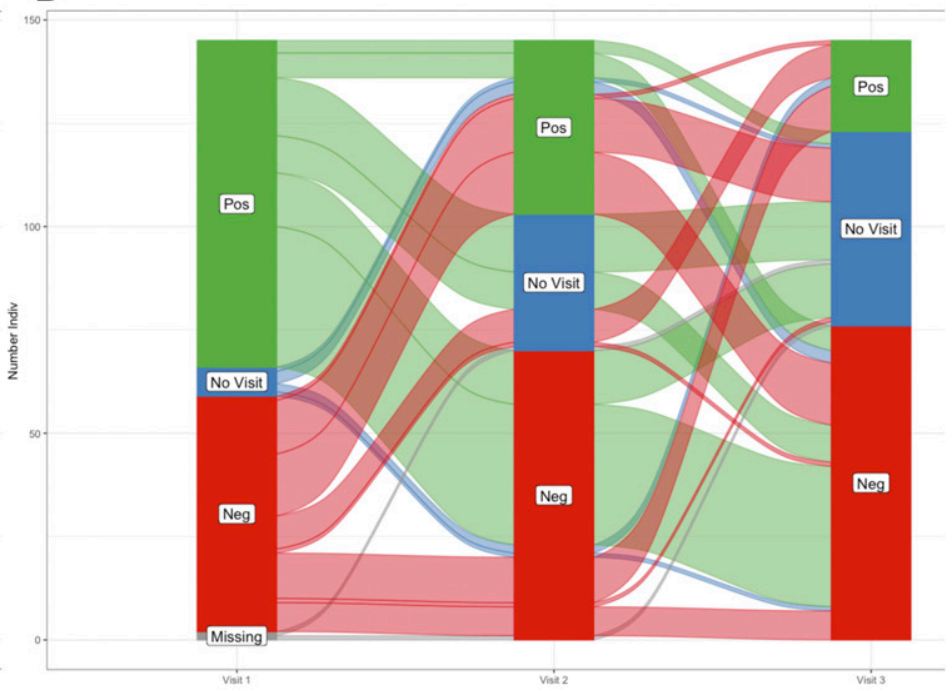

FIGURE 2. Trajectory of individual infection status spanning initial and subsequent visits. Individual test results by (A) rapid diagnostic test (RDT) and (B) quantitative PCR (qPCR) on the initial, 30-day, and 90-day visits for individuals who had at least one positive test (either by qPCR or RDT). Of these individuals, much fewer were RDT positive than those who were GPCR positive. However, in both instances, there were few consecutive positive events. This figure appears in color at www.ajtmh.org.

were all sampled in March of 2016 and were children (ages 1, 5 , and 15 years). Although the sample size was small for any statistical or robust comparisons, these results show evidence of persistent infections remaining undetected, as well as potentially new infections occurring within 1 month of reactive screen-and-treat.

\section{DISCUSSION}

Reactive screen-and-treat has been shown to be operationally and logistically challenging, and costly. ${ }^{19,23}$ Nevertheless, reactive screen-and-treat is recommended by the $\mathrm{WHO}$ as an elimination strategy where feasible in countries with low transmission ${ }^{33-35}$ and was designed to leverage the spatial and temporal clustering of malaria transmission. The programmatic goal is to identify the chronically infected reservoir that is potentially maintaining transmission in lowburden settings. This study showed that a reactive screenand-treat program could identify asymptomatic infections within index case households and provides epidemiologic evidence of focal transmission, but it also highlights the limitations of reactive screen-and-treat because of the low sensitivity of RDTs and the inability to achieve elimination.

Parasite prevalence was highest in index households at the initial visit in each season and decreased on follow-up visits but never reached zero. In 2015, Pringle et al. ${ }^{36}$ used a similar study design in the same setting but without longitudinal follow-up, and found that parasites collected closer in space and time within a single season were highly related using a panel of 26 microsatellite markers. This study included longitudinal follow-up visits and was conducted across multiple seasons. However, the 24-SNP genotyping method used in this study had lower resolution than the microsatellite method. ${ }^{36}$ Nevertheless, these findings were consistent with earlier studies that showed parasites from infections closer in space and time were more related, indicating local and focal transmission. Importantly, this study also found that parasites were related between follow-up visits and over seasons, suggesting transmission is sustained locally by persistent low parasitemic infections. Although the sample size was small, on the individual level, there was evidence of infections persisting undetected and new infections occurring between study visits, indicating the potential for sustained ongoing transmission in the presence of reactive screen-and-treat.

Parasitemia was very low in nearly all infections, with $70 \%$ falling below the limit of detection of the RDT (approximately 100 parasites $/ \mu \mathrm{L}$ ) and with a median of only 19.6 parasites $/ \mu \mathrm{L}$. This may partly explain the persistence of low-level transmission and the failure to achieve elimination despite a wellfunctioning reactive screen-and-treat program. Although the program was implemented according to national guidelines, it did not function as an efficient surveillance system to detect asymptomatic infections and reduce transmission. Even in a low-transmission setting, reactive screen-and-treat is resource intensive and falls short of achieving elimination by failing to identify low parasitemic infections. It is possible that Plasmodium falciparum histidine-rich protein 2/3 (pfhrp2/3) deletions in the parasite population could contribute to the inability of the RDT to detect infections. In this area, pfhrp2, but not pfhrp3, deletions were detected in a small sample of discordent RDT-negative/PCR-positive infections. ${ }^{37}$ However, the low parasite densities are the most likely reason for the limitations of the RDT to detect asymptomatic infections. The low parasitemia also resulted in high missingness in the genetic data, with only $46 \%$ of samples with sufficient data for analysis. We used targeted pre-amplification of parasite DNA to overcome potential bias in analyzing only samples with higher parasite densities. ${ }^{31}$ However, the high missingness highlights the challenges in conducting genetic analyses in settings with low parasitemia. The genetic analysis indicated that parasites were related within index households, at initial visits, between visits, and between seasons, suggesting these low parasitemic infections were maintained and likely transmitted into the following season. These low parasitemic infections among 
A

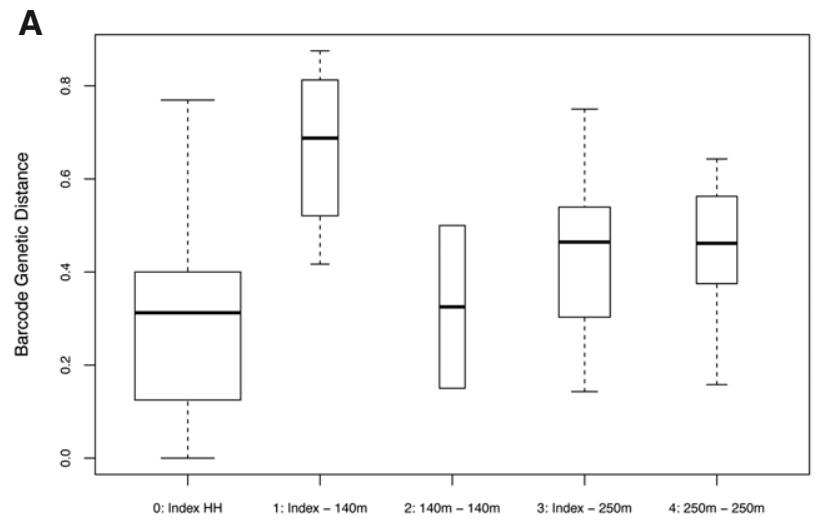

C

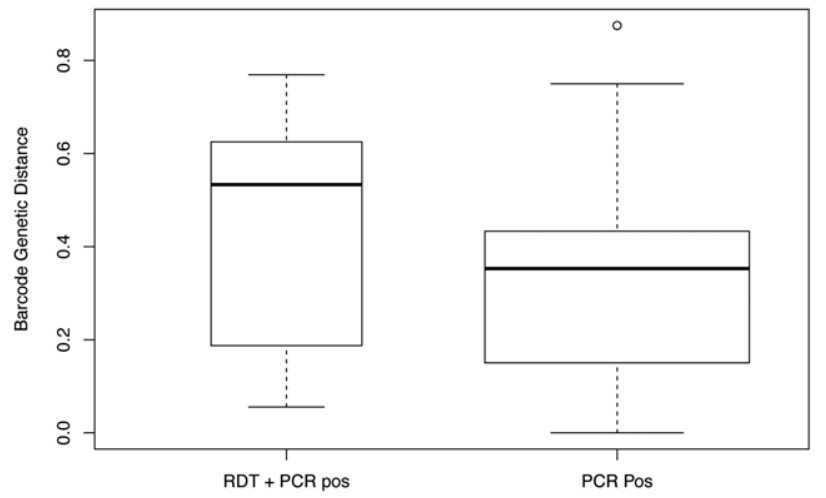

B

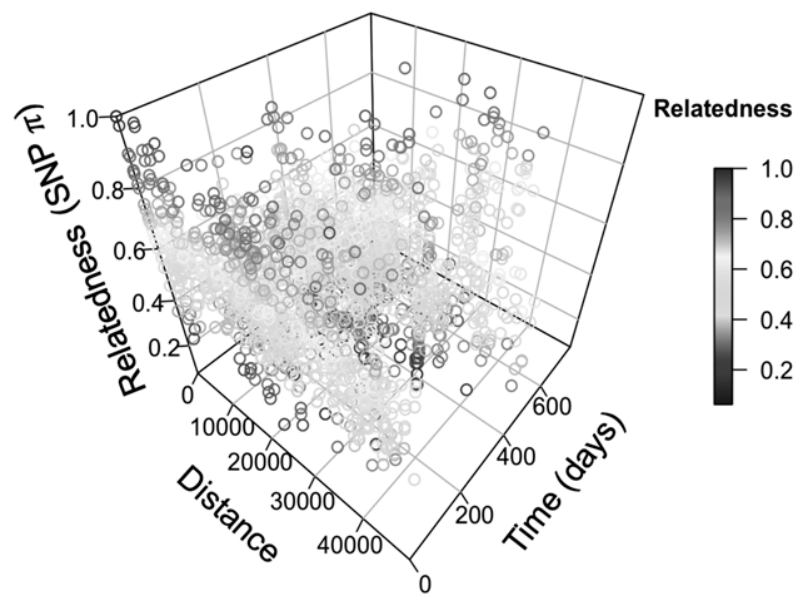

D

All Barcodes within 1 Month (2016)

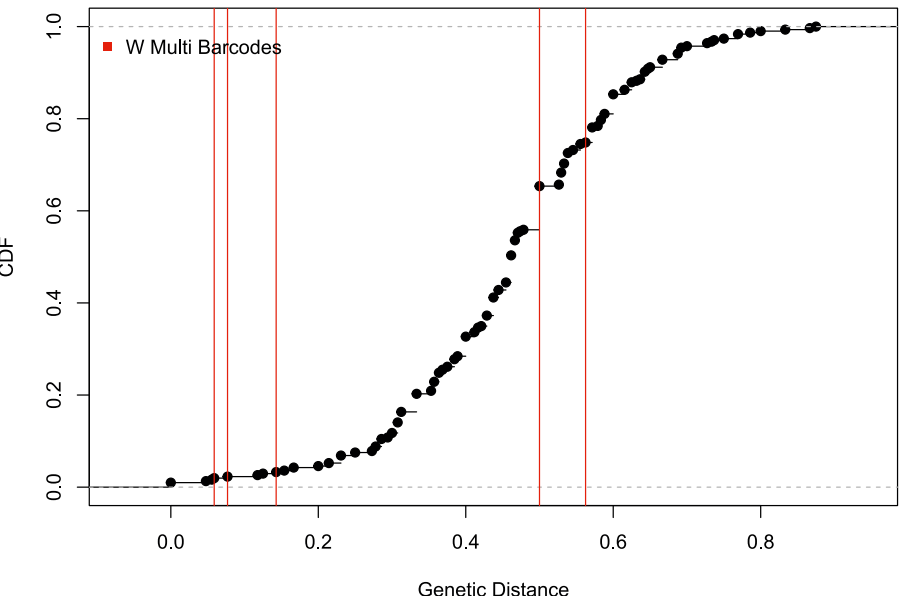

FIGURE 3. Genetic relatedness between infections. Genetic distance was calculated between infections using 24-single nucleotide polymorphism (SNP) bar codes. (A) Genetic distance of infections between all combinations of index household, households within $140 \mathrm{~m}$, and households 141-250 m of the index household was calculated. In general, infections found within the same household were the most genetically related, with no clear pattern with increasing distances or within further distances (e.g., between infections all within $140 \mathrm{~m}$ of the index household). (B) Genetic distance between all pairs of infections by physical and temporal distance was calculated with more genetically related infections shown in red. (C) All infections were compared with the index infection, when possible, based on if the secondarily identified infection was rapid diagnostic test (RDT) and quantitative PCR (qPCR) positive or only qPCR positive. Surprisingly, infections that were only qPCR positive were more related to the index infection than those that were both RDT and qPCR positive. (D) For infections that occurred within 1 month of each other, the pairwise genetic distance was calculated. Few samples with multiple bar codes (shown in redlines) all occurred within 1 month of each other and the genetic distances are shown. Cumulative distribution function of all genetic distances for the within 1 month comparison is shown. This figure appears in color at www.ajtmh.org.

the asymptomatic reservoir could be a substantial factor associated with ongoing malaria transmission in this preelimination setting. A reactive strategy should help identify foci of transmission, but when screening and treatment are based on low sensitivity RDTs, this strategy is unable to capture all parasitemic individuals. Therefore, reactive focal drug administration in index households may be more effective and efficient in eliminating transmission foci.

Many of these findings are consistent with prior findings using epidemiologic and genetic data to identify focal transmission and can inform the National Malaria Elimination Programme on how to efficiently deploy interventions to interrupt transmission and achieve malaria elimination. 9,10,12,21,22,38 Many previous studies have focused on the spatial extent and methods for screening and timing of potential follow-up visits in programs. ${ }^{21,23,39,40}$ Reactive screen-and-treat is now being implemented across several sub-Saharan African countries using similar methods as in southern Zambia., ${ }^{9,11,16}$ These have led to improved surveillance and an identification of an increased number of parasitemic individuals but have yet to successfully reduce transmission or achieve elimination. 9,23,41

Reactive screen-and-treat requires maintenance of a steady supply of large numbers of RDTs for screening and depends on community volunteers with other responsibilities. ${ }^{19}$ There are many operational challenges to follow-up in every index case, especially during the high transmission season, and not every resident is at home at the time of the home visits. ${ }^{19}$ Further efforts are needed to guide screening and increase the efficiency of reactive screen-and-treat strategies, including the use of environmental clues such as streams. ${ }^{21}$ However, even under optimal conditions, when most infections are asymptomatic, the low sensitivity of the RDT as a screening tool is the biggest challenge to the effectiveness of reactive screen-and-treat. ${ }^{19,22}$ Rapid diagnostic tests only 
identified $20 \%$ of all parasitemic individuals, which decreased over follow-up visits as parasite prevalence decreased, and is the most likely reason there transmission was not interrupted.

It is clear that malaria transmission is spatially and temporally focal, especially in pre-elimination settings. This study provides further evidence that index households represent high-risk foci for infection. This relationship can be used to identify and treat the asymptomatic reservoir, specifically through reactive focal drug administration in index households with collection of DBS for surveillance by more sensitive testing with qPCR. However, what remains is better understanding of the underlying sources of transmission within and outside these foci that are not explained by parasite relatedness or longitudinal analyses. Further investigation is warranted to fully understand these transmission dynamics and the elimination strategies built upon these findings.

Received August 3, 2020. Accepted for publication September 28, 2020.

Published online November 23, 2020.

Note: Supplemental figure appears at www.ajtmh.org.

Authors' addresses: Kelly M. Searle, Division of Epidemiology and Community Health, University of Minnesota, Minneapolis, MN, E-mail: ksearle@umn.edu. Ben Katowa, Michael Musonda, Harry Hamapumbu, Japhet Matoba, Mukuma Lubinda, Jennifer C. Stevenson, and Philip E. Thuma, Clinical Research Department, Macha Research Trust, Macha, Zambia, E-mails: ben.katowa@macharesearch.org, michael.musonda@macharesearch.org, harry.hamapumbu@macha research.org, japhet.matoba@macharesearch.org, mukuma.lubinda@ macharesearch.org, jennycstevenson80@gmail.com, and phil.thuma@ macharesearch.org. Julia C. Pringle and Douglas E. Norris, W Harry Feinstone Department of Molecular Microbiology and Immunology, Johns Hopkins Bloomberg School of Public Health, Baltimore, MD, E-mails: julia.pringle108@gmail.com and douglas.norris@jhu.edu. Timothy Shields, Tamaki Kobayashi, Amy Wesolowski, and William J. Moss, Department of Epidemiology, Johns Hopkins University Bloomberg School of Public Health, Baltimore, MD, E-mails: tshields@ jhu.edu, tkobaya2@jhu.edu, awesolowski@jhu.edu, and wmoss1@ jhu.edu.

This is an open-access article distributed under the terms of the Creative Commons Attribution (CC-BY) License, which permits unrestricted use, distribution, and reproduction in any medium, provided the original author and source are credited.

\section{REFERENCES}

1. WHO, 2018. World Malaria Report, 2018. Geneva, Switzerland: World Health Organization.

2. Gething PW et al., 2016. Mapping Plasmodium falciparum mortality in Africa between 1990 and 2015. N Engl J Med 375: 2435-2445.

3. Kobayashi T et al., 2019. Characteristics of subpatent malaria in a pre-elimination setting in southern Zambia. Am J Trop Med Hyg 100: 280-286.

4. Bousema T, Okell L, Felger I, Drakeley C, 2014. Asymptomatic malaria infections: detectability, transmissibility and public health relevance. Nat Rev Microbiol 12: 833-840.

5. Lindblade KA, Steinhardt L, Samuels A, Kachur SP, Slutsker L, 2013. The silent threat: asymptomatic parasitemia and malaria transmission. Expert Rev Anti Infect Ther 11: 623-639.

6. Bousema T, Griffin JT, Sauerwein RW, Smith DL, Churcher TS, Takken W, Ghani A, Drakeley C, Gosling R, 2012. Hitting hotspots: spatial targeting of malaria for control and elimination. PLoS Med 9: e1001165.

7. Bjorkman A, Cook J, Sturrock H, Msellem M, Ali A, Xu W, Molteni $\mathrm{F}$, Gosling R, Drakeley $\mathrm{C}$, Mårtensson A, 2017. Spatial distribution of falciparum malaria infections in Zanzibar: implications for focal drug administration strategies targeting asymptomatic parasite Carriers. Clin Infect Dis 64: 1236-1243.

8. Stresman GH et al., 2015. Focal screening to identify the subpatent parasite reservoir in an area of low and heterogeneous transmission in the Kenya highlands. J Infect Dis 212: 1768-1777.

9. Aidoo EK et al., 2018. Reactive case detection of Plasmodium falciparum in western Kenya highlands: effective in identifying additional cases, yet limited effect on transmission. Malar J 17: 111.

10. Bridges DJ, Miller JM, Chalwe V, Moonga H, Hamainza B, Steketee R, Silumbe K, Nyangu J, Larsen DA, 2017. Community-led responses for elimination (CoRE): a study protocol for a community randomized controlled trial assessing the effectiveness of community-level, reactive focal drug administration for reducing Plasmodium falciparum infection prevalence and incidence in Southern Province, Zambia. Trials 18: 511.

11. Gerardin J, Bever CA, Bridenbecker D, Hamainza B, Silumbe K, Miller JM, Eisele TP, Eckhoff PA, Wenger EA, 2017. Effectiveness of reactive case detection for malaria elimination in three archetypical transmission settings: a modelling study. Malar $J$ 16: 248.

12. Gerardin J, Bever CA, Hamainza B, Miller JM, Eckhoff PA, Wenger EA, 2016. Optimal population-level infection detection strategies for malaria control and elimination in a spatial model of malaria transmission. PLoS Comput Biol 12: e1004707.

13. Ruktanonchai NW, DeLeenheer $P$, Tatem AJ, Alegana VA, Caughlin TT, Zu Erbach-Schoenberg E, Lourenço C, Ruktanonchai CW, Smith DL, 2016. Identifying malaria transmission foci for elimination using human mobility data. PLoS Comput Biol 12: e1004846.

14. Larsen DA et al., 2015. Malaria surveillance in low-transmission areas of Zambia using reactive case detection. Malar J 14: 465.

15. Larson BA, Ngoma T, Silumbe K, Rutagwera MR, Hamainza B, Winters AM, Miller JM, Scott CA, 2016. A framework for evaluating the costs of malaria elimination interventions: an application to reactive case detection in Southern Province of Zambia Malar J 15: 408.

16. Littrell M, Sow GD, Ngom A, Ba M, Mboup BM, Dieye Y, Mutombo $B$, Earle D, Steketee RW, 2013. Case investigation and reactive case detection for malaria elimination in northern Senegal. Malar J 12: 331.

17. Hustedt J et al., 2016. Reactive case-detection of malaria in Pailin Province, Western Cambodia: lessons from a year-long evaluation in a pre-elimination setting. Malar J 15: 132.

18. Medzihradsky OF et al., 2018. Study protocol for a cluster randomised controlled factorial design trial to assess the effectiveness and feasibility of reactive focal mass drug administration and vector control to reduce malaria transmission in the low endemic setting of Namibia. BMJ Open 8: e019294.

19. Searle KM et al., 2016. Evaluation of the operational challenges in implementing reactive screen-and-treat and implications of reactive case detection strategies for malaria elimination in a region of low transmission in southern Zambia. Malar J 15: 412.

20. Mharakurwa S, Thuma PE, Norris DE, Mulenga M, Chalwe V, Chipeta J, Munyati S, Mutambu S, Mason PR; Southern Africa ICEMR Team, 2012. Malaria epidemiology and control in southern Africa. Acta Trop 121: 202-206.

21. Bhondoekhan FRP et al., 2020. Improving the efficiency of reactive case detection for malaria elimination in southern Zambia: a cross-sectional study. Malar J 19: 175.

22. Deutsch-Feldman $\mathrm{M}$ et al., 2018. Efficiency of a malaria reactive test-and-treat program in southern Zambia: a prospective, observational study. Am J Trop Med Hyg 98: 1382-1388.

23. Sturrock HJ, Novotny JM, KuneneS, Dlamini S, Zulu Z, Cohen JM, Hsiang MS, Greenhouse B, Gosling RD, 2013. Reactive case detection for malaria elimination: real-life experience from an ongoing program in Swaziland. PLoS One 8: e63830.

24. Moss WJ, Hamapumbu H, Kobayashi T, Shields T, Kamanga A, Clennon J, Mharakurwa S, Thuma PE, Glass G, 2011. Use of remote sensing to identify spatial risk factors for malaria in a region of declining transmission: a cross-sectional and longitudinal community survey. Malar $J$ 10: 163. 
25. Kent RJ, Thuma PE, Mharakurwa S, Norris DE, 2007. Seasonality, blood feeding behavior, and transmission of Plasmodium falciparum by Anopheles arabiensis after an extended drought in southern Zambia. Am J Trop Med Hyg 76: 267-274.

26. Laban NM, Kobayashi T, Hamapumbu H, Sullivan D, Mharakurwa S, Thuma PE, Shiff CJ, Moss WJ; Southern Africa International Centers of Excellence for Malaria Research, 2015. Comparison of a PfHRP2-based rapid diagnostic test and PCR for malaria in a low prevalence setting in rural southern Zambia: implications for elimination. Malar J 14: 25.

27. Sipilanyambe N, Simon JL, Chanda P, Olumese P, Snow RW, Hamer DH, 2008. From chloroquine to artemetherlumefantrine: the process of drug policy change in Zambia. Malar J 7: 25.

28. Steketee RW, Sipilanyambe N, Chimumbwa J, Banda JJ, Mohamed A, Miller J, Basu S, Miti SK, Campbell CC, 2008. National malaria control and scaling up for impact: the Zambia experience through 2006. Am J Trop Med Hyg 79: 45-52.

29. Daniels $R$ et al., 2008. A general SNP-based molecular barcode for Plasmodium falciparum identification and tracking. Malar $\mathrm{J}$ 7: 223.

30. Searle KM et al., 2017. Distinct parasite populations infect individuals identified through passive and active case detection in a region of declining malaria transmission in southern Zambia. Malar J 16: 154.

31. Mharakurwa S, Daniels R, Scott A, Wirth DF, Thuma $P$, Volkman SK, 2014. Pre-amplification methods for tracking low-grade Plasmodium falciparum populations during scaled-up interventions in Southern Zambia. Malar J 13: 89.

32. Ippolito MM, Searle KM, Hamapumbu H, Shields TM, Stevenson JC, Thuma PE, Moss WJ; For The Southern Africa International Center Of Excellence For Malaria Research, 2017. House structure is associated with Plasmodium falciparum infection in a low-transmission setting in southern Zambia. Am J Trop Med Hyg 97: 1561-1567.

33. WHO, 2012. Disease Surveillance for Malaria Elimination: an Operational Manual. Geneva, Switzerland: World Health Organization.

34. WHO, 2018. World Malaria Report. Geneva, Switzerland: World Health Organization.

35. WHO Programme, 2017. A Framework for Malaria Elimination. Geneva, Switzerland: World Health Organization.

36. Pringle JC et al., 2018. Genetic evidence of focal Plasmodium falciparum transmission in a pre-elimination setting in southern province, Zambia. J Infect Dis 219: 1254-1263.

37. Kobayashi T et al., 2019. The search for Plasmodium falciparum histidine-rich protein 2/3 deletions in Zambia and implications for Plasmodium falciparum histidine-rich protein 2-based rapid diagnostic tests. Am J Trop Med Hyg 100: 842-845.

38. Grossenbacher B, Holzschuh A, Hofmann NE, Abdullah Omar KA, Stuck L, Shariff Fakih B, Ali A, Yukich J, Hetzel MW, Felger I, 2020. Molecular methods for tracking residual Plasmodium falciparum transmission in a close-to-elimination setting in Zanzibar. Malar J 19: 50.

39. Searle KM, Shields T, Hamapumbu H, Kobayashi T, Mharakurwa S, Thuma PE, Smith DL, Glass G, Moss WJ, 2013. Efficiency of household reactive case detection for malaria in rural Southern Zambia: simulations based on cross-sectional surveys from two epidemiological settings. PLoS One 8: e70972.

40. Nelli L, Guelbeogo M, Ferguson HM, Ouattara D, Tiono A, N'Fale S, Matthiopoulos J, 2020. Distance sampling for epidemiology: an interactive tool for estimating under-reporting of cases from clinic data. Int J Health Geogr 19: 16.

41. van Eijk AM et al., 2016. What is the value of reactive case detection in malaria control? A case-study in India and a systematic review. Malar J 15: 67. 